\title{
Multiple scattering by cylinders immersed in fluid: high order approximations for the effective wavenumbers
}

\author{
Andrew N. Norria \\ Mechanical 83 Aerospace Engineering, Rutgers University, Piscataway, NJ 08854, USA \\ Jean-Marc Conoir \\ Institut Jean Le Rond d'Alembert, UPMC Univ Paris 06, UMR 7190, F-75005 Paris, France
}

(Dated: December 14, 2018)

\begin{abstract}
Acoustic wave propagation in a fluid with a random assortment of identical cylindrical scatterers is considered. While the leading order correction to the effective wavenumber of the coherent wave is well established at dilute areal density $\left(n_{0}\right)$ of scatterers, in this paper the higher order dependence of the coherent wavenumber on $n_{0}$ is developed in several directions. Starting from the quasicrystalline approximation (QCA) a consistent method is described for continuing the Linton and Martin formula, which is second order in $n_{0}$, to higher orders. Explicit formulas are provided for corrections to the effective wavenumber up to $\mathrm{O}\left(n_{0}^{4}\right)$. Then, using the QCA theory as a basis, generalized self consistent schemes are developed and compared with self consistent schemes using other dynamic effective medium theories. It is shown that the Linton and Martin formula provides a closed self-consistent scheme, unlike other approaches.
\end{abstract}

PACS numbers: 43.35Bf, 43.20.Fn, 43.20.Hq

\section{INTRODUCTION}

It is often assumed that multiple scattering by a dilute array of scatterers in a perfect fluid may be described by the propagation of a coherent wave representing the acoustic field averaged over all possible scatterer configurations. The coherent wave has a complex-valued wavenumber, $k_{e f f}$, often called the effective wavenumber. Its imaginary part accounts for loss due to scattering in all directions. Foldy first derived an expression for the effective wavenumber of point scatterers (isotropic scattering $)^{1}$. Subsequently, Waterman and Truell considered finite size scatterers (non-isotropic scattering $)^{2}$ and obtained a second order correction to Foldy's formula in terms of the scatterer density. The averaged exciting field $\mathrm{in}^{2}$ is obtained by replacing the spherical scatterers by point scatterers with the same angle-dependent far-field scattering amplitude as the actual finite size scatterers. For cylindrical scatterers, Waterman and Truell's approach provides $k_{e f f}=k_{W T}$ where ${ }^{3}$

$$
k_{W T}^{2}=\left[k-\frac{2 i n_{0}}{k} f(0)\right]^{2}-\left[\frac{2 i n_{0}}{k} f(\pi)\right]^{2},
$$

\footnotetext{
a) Electronic address: norris@rutgers.edu
}

with $f(\theta)$, defined in $₫$, the far-field scattered amplitude in direction $\theta$ for each cylinder and $n_{0}$ the number of scatterers per unit area.

In 1967 Lloyd and Berry ${ }^{4}$ proposed an explicit expression for spherical scatterers different from that of Waterman and Truell. While Lloyd and Berry's formula had been obtained for spherical scatterers by using a different method ${ }^{5}$, Linton and Martin derived its counterpart for cylindrical scatterers in 2005흐. Linton and Martin's formula may be recovered from Fikioris and Waterman's dispersion relation $\underline{7}$ by expanding it in powers of $n_{0}$, under the assumption that $n_{0} / k^{2}<<1$, and by letting the radius of exclusion in the hole correction tends to zero. For cylindrical scatterers, Linton and Martin's approach yields $k_{e f f}=k_{L M}$,

$k_{L M}^{2}=k^{2}-4 i n_{0} f(0)+\frac{8 n_{0}^{2}}{\pi k^{2}} \int_{0}^{\pi} \cot \left(\frac{\theta}{2}\right) \frac{\mathrm{d}}{\mathrm{d} \theta}[f(\theta)]^{2} \mathrm{~d} \theta$.

This formula is only valid for symmetric scattering functions satisfying $f(\theta)=f(-\theta)$. Its generalization to arbitrary $f$ is obtained by replacing $f^{2}(\theta)$ with $f(\theta) f(-\theta)$ in (2), see below and ${ }^{8}$. Linton and Martin's formula is a consequence of the widely used closure assumption known as the quasi-crystalline approximation (QCA). Martin and Maure ${ }^{9}$ demonstrated that QCA agrees with the Lippmann-Schwinger approach (weak scatter- 
ing) to $\mathrm{O}\left(n_{0}^{2}\right)$.

The Independent Scattering Approximation (ISA) $\stackrel{10}{\Perp}$ is a simplistic approximation in multiplescattering theory, sometimes used without justification. It can be deduced directly from Eq. (11) and Eq. (2) by neglecting the term of second order in $n_{0} / k^{2}$, giving $k_{e f f}=k_{I S A}$ with

$$
k_{I S A}^{2}=k^{2}-4 i n_{0} f(0) .
$$

All of these methods are explicit since $k_{\text {eff }}$ is given by a formula. But the question remains: which method is the most accurate? This depends largely on the value of the concentration, $c=n_{0} \pi a^{2}$, where $a$ is the radius for circular cylinders or the radius of the enclosing cylinder for noncircular scatterers. It is evident that ISA, which is a method of order one in $n_{0} / k^{2}=c / \pi(k a)^{2}$, is the least precise. As for the two others, according to the analysis made by Linton and Martin in ${ }^{\underline{6}}$, it is justified to think that $k_{L M}$ is more accurate than $k_{W T}$. However, the difference between $k_{W T}$ and $k_{L M}$ is small at low concentration, as shown in 11 when $c=6 \%$. Ref $\underline{11}$ also shows that the Waterman and Truell method or ISA fail while Linton and Martin's gives better results for higher concentration: $c=14 \%$.

There are two other methods that deal with the far field scattered amplitudes, but they are implicit; $k_{\text {eff } f}$ is obtained by solving an equation. The most famous is the Coherent Potential Approximation (CPA), and its generalization (GCPA), both based on Dyson's equation ${ }^{12}$. There is also the Generalized Self Consistent Model (GSCM) which is derived using a self consistent scheme applied to Waterman and Truell's formula 13 . The existence of a non trivial solution to the homogeneous system of linear equations obtained by Fikioris and Waterman also allows one to calculate a $k_{\text {eff }} \underline{14}$. According to the finding of Cowan et al.$^{15}$ the CPA is capable of accounting for concentrations up to $60 \%$ for glass beads in a liquid mixture of water and glycerol. Of course, scatterers do not radiate as much at this level of concentration as compared to those considered at the lower levels of concentration in $\underline{11}$. Nevertheless, the results of $\frac{15}{15}$ suggest that implicit methods can be more powerful than explicit ones even if, to our knowledge, no rigorous comparison has been made between the two types of methods. Ref $\frac{16}{}$ provides comprehensive numerical comparisons of eight different explicit and implicit methods.

In this paper we search for explicit and implicit expressions of $k_{\text {eff }}$ that could allow us to consider higher concentrations for multiple scatter- ing problems. For explicit methods, the only way to improve $k_{\text {eff }}$ is to extend Linton and Martin's formula to orders higher than 2 in concentration. This is accomplished in that Waterman and Truell's formula is of order 2 in concentration, and that it is is not possible to go further. For implicit methods we use the self consistent scheme as used by Yang and Mal in 13 . When applied to the ISA this self consistent scheme leads to CPA. It is applied to the generalized Linton and Martin formula in $₫[\mathrm{IV}$ where the physical meaning of the self consistent scheme is discussed. In particular, we obtain a new result that generalizes the CPA. In $₫ \mathrm{~V}$ we compare all the effective wavenumbers in the Rayleigh limit (low frequencies).

\section{HIGHER ORDER THEORY}

\section{A. Multiple scattering formulation}

The problem is formulated in terms of the pressure $\psi(\vec{r})$ which satisfies the Helmholtz equation in the interstitial space between scatterers:

$$
\nabla^{2} \psi+k^{2} \psi=0
$$

where $k=\omega / c$ and $c$ is the speed of sound. Time harmonic dependence $e^{-i \omega t}$ is assumed. Consider first a system of $N$ scatterers with fixed positions, for which the total field can be expressed

$$
\psi(\vec{r})=\psi_{i n c}(\vec{r})+\sum_{j=1}^{N} T\left(\vec{r}_{j}\right) \psi_{E}\left(\vec{r}, \vec{r}_{j}\right) .
$$

Here, $\psi_{\text {inc }}$ is the incident wave, $\psi_{E}\left(\vec{r}, \vec{r}_{j}\right)$ is the exciting field for scatterer $j$, and $T\left(\vec{r}_{j}\right)$ its scattering operator. Focusing on scatterer $j=1$ as representative, we have

$$
\psi_{E}\left(\vec{r}, \vec{r}_{1}\right)=\psi_{i n c}(\vec{r})+\sum_{j=2}^{N} T\left(\vec{r}_{j}\right) \psi_{E}\left(\vec{r}, \vec{r}_{j}\right) .
$$

If, as is the case here, the positions are not known, it becomes necessary to assume some type of statistical description. The number density $n$ for $N$ discrete scatterers with centers at $\vec{r}_{1}, \vec{r}_{2} \ldots$, is $n\left(\vec{r}_{1}, \vec{r}_{2}, \ldots, \vec{r}_{N}\right)=N p\left(\vec{r}_{1}, \vec{r}_{2}, \ldots, \vec{r}_{N}\right)$, where $p$ is the probability density. Conditional densities, defined by fixing the position of one scatterer, satisfy 2 $n\left(\vec{r}_{2}, \ldots, \vec{r}_{N} \mid \vec{r}_{1}\right)=(N-1) p\left(\vec{r}_{2}, \ldots, \vec{r}_{N} \mid \vec{r}_{1}\right)$. For our purposes we need only the conditional number density of the $j^{\text {th }}$ scatterer if a scatterer is known to 
be at $\vec{r}_{1}: n\left(\vec{r}_{j} \mid \vec{r}_{1}\right)=(N-1) p\left(\vec{r}_{j} \mid \vec{r}_{1}\right), \quad j \neq 1$, satisfying $\int \mathrm{d} \vec{r}_{j} n\left(\vec{r}_{j} \mid \vec{r}_{1}\right)=N-1$. The analog of (6) is then an equation for the configurationally averaged field, $\left\langle\psi_{E}\left(\vec{r} \mid \vec{r}_{1}\right)\right\rangle$,

$$
\begin{aligned}
& \left\langle\psi_{E}\left(\vec{r} \mid \vec{r}_{1}\right)\right\rangle=\psi_{i n c}(\vec{r}) \\
& \quad+\int \mathrm{d} \vec{r}_{j} n\left(\vec{r}_{j} \mid \vec{r}_{1}\right) T\left(\vec{r}_{j}\right)\left\langle\psi_{E}\left(\vec{r} \mid \vec{r}_{j}, \vec{r}_{1}\right)\right\rangle .
\end{aligned}
$$

As noted $b^{2}{ }^{2}$, "The fact that the exciting field with one scatterer fixed is given in terms of the field with two scatterers fixed is the basic difficulty in multiple scattering". We adopt perhaps the simplest solution to this quandary, the QCA (quasicrystalline approximation), under which assumption (7) reduces to

$$
\begin{aligned}
& \left\langle\psi_{E}\left(\vec{r} \mid \vec{r}_{1}\right)\right\rangle=\psi_{\text {inc }}(\vec{r}) \\
& \quad+\int \mathrm{d} \vec{r}_{j} n\left(\vec{r}_{j} \mid \vec{r}_{1}\right) T\left(\vec{r}_{j}\right)\left\langle\psi_{E}\left(\vec{r} \mid \vec{r}_{j}\right)\right\rangle .
\end{aligned}
$$

The scattering operator for every scatterer is assumed to have translational invariance with

$$
\begin{aligned}
T(\overrightarrow{0}) e^{i k x} & =\sum_{n} i^{n} T_{n} H_{n}^{(1)}(k r) e^{i n \theta} \\
& \stackrel{\simeq}{\rightarrow} \infty) \sqrt{\frac{2}{\pi k r}} e^{i\left(k r-\frac{\pi}{4}\right)} f(\theta),
\end{aligned}
$$

and therefore the Fourier series for $f$ is

$$
f(\theta)=\sum_{n} T_{n} e^{i n \theta}
$$

The assumed form of the operator in (9) means that each scatterer has the same scattering behavior. We consider plane wave incidence, $\psi_{\text {inc }}(\vec{r})=$ $A e^{i k x}$. The exciting potential $\left\langle\psi_{E}\left(\vec{r}, \vec{r}_{j}\right)\right\rangle$ satisfies the Helmholtz equation and is regular function at the point $\overrightarrow{r_{j}}$, it can therefore be expressed

$$
\left\langle\psi_{E}\left(\vec{r} \mid \vec{r}_{j}\right)\right\rangle=\sum_{n} A_{n}\left(\vec{r}_{j}\right) J_{n}\left(k \rho_{j}\right) e^{i n \theta\left(\vec{\rho}_{j}\right)},
$$

where $\vec{\rho}_{j}=\vec{r}-\vec{r}_{j}, \theta\left(\vec{\rho}_{j}\right)=\arg \left(\vec{\rho}_{j}\right)$. Substituting from (11) into the configurational average equation (8) and using the addition theorem for cylinder functions ${ }^{8}$, yields

$$
\begin{gathered}
A_{n}\left(\vec{r}_{1}\right)=i^{n} e^{i k x_{1}}+\sum_{p}(-1)^{p} T_{n+p} \int_{S^{+}} n\left(\vec{r}_{j} \mid \vec{r}_{1}\right) \\
\times A_{n+p}\left(\vec{r}_{j}\right) H_{n}^{(1)}\left(k \rho_{j}\right) e^{i n \theta\left(\vec{\rho}_{j}\right)} \mathrm{d} \vec{r}_{j},
\end{gathered}
$$

on the half-space $S^{+}=\{x>0\}$. The effective wavenumber $\xi$ defines the coherent wave according to the assumed functional form for each $A_{n}$ :

$$
A_{n}\left(\vec{r}_{j}\right)=i^{n} A_{n} e^{i \xi x_{j}} .
$$

Use of the addition theorem for cylindrical functions then implies

$$
A_{n} e^{i \xi x_{1}}=A e^{i k x_{1}}+\sum_{p} T_{n+p} A_{n+p} L_{p}(\xi)
$$

with

$$
\begin{aligned}
L_{p}(\xi)=i^{-p} & \int_{S^{+}} \mathrm{d} \vec{r}_{j} n\left(\vec{r}_{j} \mid \vec{r}_{1}\right) \\
& \times H_{p}^{(1)}\left(k r_{j 1}\right) e^{i p \theta\left(\vec{r}_{j 1}\right)} e^{i \xi x_{j}},
\end{aligned}
$$

where $\vec{r}_{j 1}=\vec{r}_{j}-\vec{r}_{1}, \theta\left(\vec{r}_{j 1}\right)=\arg \left(\vec{r}_{j 1}\right)$.

We assume a modified form of the hole correction with hole radius $b$,

$$
n\left(\vec{r}_{j} \mid \vec{r}_{1}\right)= \begin{cases}n_{0}\left(1+h\left(r_{j 1}\right)\right), & r_{j 1}>b, \\ 0, & r_{j 1} \leq b,\end{cases}
$$

where $h$ represents the deviation from the constant background value. It satisfies $h(r) \rightarrow 0$ as $r \rightarrow \infty$, with the stronger condition

$$
\lim _{R \rightarrow \infty} R^{-2} \int_{b}^{R} h(r) r \mathrm{~d} r=0 .
$$

Following, e.g. $\stackrel{8}{ }$, it may be shown that the integral (15) reduces to

$$
\begin{aligned}
L_{p}(\xi)= & 2 \pi n_{0}\left\{\frac{N_{p}(\xi b)}{\xi^{2}-k^{2}}+\frac{M_{p}(\xi b)}{k^{2}}\right\} e^{i \xi x_{1}} \\
& +\frac{2 i n_{0}}{k(\xi-k)} e^{i k x_{1}}
\end{aligned}
$$

where

$$
\begin{aligned}
& N_{p}(\xi b)=\xi b J_{p}^{\prime}(\xi b) H_{p}^{(1)}(k b)-k b J_{p}(\xi b) H_{p}^{(1)^{\prime}}(k b), \\
& M_{p}(\xi b)=\int_{b}^{\infty} J_{p}(\xi r) H_{p}^{(1)}(k r) h(r) k^{2} r \mathrm{~d} r .
\end{aligned}
$$

The simple "hole correction" is $h=0$ and hence $M_{p}=0$; see Sec. IV.D of Ref $\stackrel{6}{\underline{6} \text { for further discus- }}$ sion of the case $h \neq 0$.

\section{B. Matrix formulation}

Substituting from (18) into (14) and equating to zero the coefficients of $e^{i \zeta x_{1}}$ and $e^{i k x_{1}}$ yields two equations, known as the Lorentz-Lorenz law and the extinction theorem respectively,

$$
\begin{aligned}
A_{n}+\frac{2 \pi n_{0}}{k^{2}-\xi^{2}} \sum_{p=-\infty}^{\infty} \mathcal{N}_{n-p}(\xi b) T_{p} A_{p} & =0, \\
A+\frac{2 i n_{0}}{k(\xi-k)} & \sum_{p=-\infty}^{\infty} T_{p} A_{p}=0
\end{aligned}
$$


where (20a) is satisfied by all $n \in \mathbb{Z}$ and

$$
\mathcal{N}_{p}(\xi b)=N_{p}(\xi b)+\left(\frac{\xi^{2}}{k^{2}}-1\right) M_{p}(\xi b) .
$$

Equation (20a) is a homogeneous system which defines the effective wavenumber and the associated infinite eigenvector, while Eq. (20b) defines the amplitude of the eigenvector in terms of the excitation amplitude $A$. The identity $\mathcal{N}_{-p}(\xi)=\mathcal{N}_{p}(\xi)$ follows from the known properties of Bessel and Hankel functions. Equation (20a) with $h=0 \Rightarrow$ $M_{p}=0$ is equivalent to the system of equations studied by Linton-Martin $\underline{\underline{6}}$ (their equation (71)). We focus on the solution of Eq. (20a).

We introduce non-dimensional parameters $y$ and $\epsilon$ which depend on $b$, along with some related vectors and matrices:

$$
\begin{aligned}
y & =(\xi b)^{2}-(k b)^{2}, \\
\epsilon & =-4 i n_{0} b^{2}, \\
\mathbf{b} & =\mathbf{T}^{1 / 2} \mathbf{a}, \\
\mathbf{u} & =\mathbf{T}^{1 / 2} \mathbf{e}, \\
\mathbf{Q}(y) & =(k b)^{-2} \mathbf{T}^{1 / 2} \overline{\mathbf{Q}} \mathbf{T}^{1 / 2},
\end{aligned}
$$

where the vectors $\mathbf{a}$ and $\mathbf{e}$, the diagonal matrix $\mathbf{T}$, and the symmetric matrix $\overline{\mathbf{Q}}$, are defined

$$
\begin{aligned}
\mathbf{a} & =\left(\ldots, A_{-1}, A_{0}, A_{1}, \ldots\right)^{t}, \\
\mathbf{e} & =(\ldots, 1,1,1, \ldots)^{t}, \\
T_{m n} & =T_{n} \delta_{m n} \\
\bar{Q}_{m n} & =\frac{\frac{i \pi}{2} \mathcal{N}_{m-n}(\xi b)-1}{(\xi / k)^{2}-1},
\end{aligned}
$$

Then Eq. 20a can be expressed

$$
\left\{y(\mathbf{I}-\epsilon \mathbf{Q}(y))-\epsilon \mathbf{u u}^{t}\right\} \mathbf{b}=0 .
$$

Setting the determinant of the matrix $\{.$.$\} of this$ infinite system of equations yields the desired dispersion relation for $\xi$. We next reduce this to a simple and transparent form, and obtain an expression for the amplitude vector $\mathbf{a}$.

\section{Implicit solutions for $\xi$ and a}

Multiply (24) from the left by the inverse of (I$\epsilon \mathbf{Q})$, yielding

$$
\left(\mathbf{I}+\mathbf{w} \mathbf{u}^{t}\right) \mathbf{b}=0
$$

with infinite vector $\mathbf{w}=-\epsilon y^{-1}(\mathbf{I}-\epsilon \mathbf{Q})^{-1} \mathbf{u}$. Noting that $\operatorname{det}\left(\mathbf{I}+\mathbf{w} \mathbf{u}^{t}\right)=1+\mathbf{w}^{t} \mathbf{u}$, we deduce that the solution for $y$ can be expressed implicitly as

$$
y=\epsilon \mathbf{u}^{t}[\mathbf{I}-\epsilon \mathbf{Q}(y)]^{-1} \mathbf{u} .
$$

The dependence upon $b$ in Eq. (26) may be removed by introducing alternative non-dimensional scalars $\bar{y}=y /(k b)^{2}$ and $\bar{\epsilon}=\epsilon /(k b)^{2}$, that is,

$$
\bar{y}=\xi^{2} k^{-2}-1, \quad \bar{\epsilon}=-4 i n_{0} k^{-2},
$$

and writing $\overline{\mathbf{Q}}=\overline{\mathbf{Q}}(\bar{y})$, in terms of which Eq. 26 becomes

$$
\bar{y}=\mathbf{e}^{t}\left[(\bar{\epsilon} \mathbf{T})^{-1}-\overline{\mathbf{Q}}(\bar{y})\right]^{-1} \mathbf{e} .
$$

This formula clearly splits the dependence upon the scattering matrix $\mathbf{T}$, from that of multiple interactions, $\mathbf{Q}$. The choice of the symmetric matrix $\overline{\mathbf{Q}}$, and hence $\mathbf{Q}$, is motivated by the observation that at leading order the elements $\mathcal{N}_{n}$ are equal: $\mathcal{N}_{n}(k b)=2 /(i \pi)$ for all $n$. Despite the apparent pole at $\xi=k$ in Eq. (23d), the matrix $\overline{\mathbf{Q}}(\bar{y})$ is a regular function of $\bar{y}$ at the origin since the limit and its derivatives exist as $\xi \rightarrow k$. The solution of Eq. (20a) may be expressed in implicit form through either of the identities (26) or (28). We will find both useful in different circumstances. The latter is simpler for considering general properties, while the former is useful for the particular limit as $b \rightarrow 0$.

The identity $1+\mathbf{w}^{t} \mathbf{u}=0$ also implies that the null vector of (25) is of the form $\mathbf{b}=\alpha \mathbf{w}(\alpha \neq 0)$. The precise value of $\alpha$ follows from the extinction theorem (20b), and the vector of amplitudes $A_{n}$ can then be expressed as

$$
\mathbf{a}=\frac{2 k}{k+\xi}[(\mathbf{I}-\bar{\epsilon} \overline{\mathbf{Q}}(\bar{y}) \mathbf{T})]^{-1} \mathbf{e} .
$$

\section{Asymptotic expansion}

We seek an asymptotic expansion of $y=y_{\epsilon}$ in powers of the small parameter $\epsilon$ :

$$
y_{\epsilon}=\epsilon y_{1}+\epsilon^{2} y_{2}+\epsilon^{3} y_{3}+\ldots .
$$

The individual terms follow from (26) as

$$
y_{n}=\left.\frac{1}{n !} \mathbf{u}^{t} \frac{\mathrm{d}^{n}}{\mathrm{~d} \epsilon^{n}}\left\{\epsilon\left[\mathbf{I}-\epsilon \mathbf{Q}\left(y_{\epsilon}\right)\right]^{-1}\right\}\right|_{\epsilon=0} \mathbf{u} .
$$

Using the expansion $(1-x)^{-1}=1+x+x^{2}+\ldots$ in (31) and noting that the derivative is evaluated 
at $\epsilon=0$ means that only a finite number of terms are necessary for a given $n$. Thus,

$$
\begin{aligned}
& y_{n}=\frac{1}{n !} \mathbf{u}^{t} \frac{\mathrm{d}^{n}}{\mathrm{~d} \epsilon^{n}}\left\{\epsilon^{n} \mathbf{Q}^{n-1}(0)+\epsilon^{n-1} \mathbf{Q}^{n-2}\left(\epsilon y_{1}\right)\right. \\
& \left.\ldots+\epsilon^{2} \mathbf{Q}\left(\epsilon y_{1}+\ldots+\epsilon^{n-2} y_{n-2}\right)\right\}\left.\right|_{\epsilon=0} \mathbf{u} .
\end{aligned}
$$

We only need to expand each term in this finite series to obtain its $\mathrm{O}\left(\epsilon^{n}\right)$ contribution. This is simple for the first term $\epsilon^{n} \mathbf{Q}^{n}(0)$, and for the second it is $\epsilon^{n-1} \mathbf{Q}^{n}\left(\epsilon y_{1}\right) \rightarrow \epsilon^{n} y_{1} \frac{\mathrm{d}}{\mathrm{d} y} \mathbf{Q}^{n-2}(0)$ where $\left.\frac{\mathrm{d}}{\mathrm{d} y} g(0) \equiv \frac{\mathrm{d}}{\mathrm{d} y} g(y)\right|_{y=0}$. Subsequent terms become more complicated but the procedure for finding their contribution is straightforward. Thus,

$$
\begin{aligned}
y_{n}=\mathbf{u}^{t} & \left\{\mathbf{Q}^{n-1}(0)+y_{1} \frac{\mathrm{d}}{\mathrm{d} y} \mathbf{Q}^{n-2}(0)\right. \\
+ & \frac{y_{1}^{2}}{2} \frac{\mathrm{d}^{2}}{\mathrm{~d} y^{2}} \mathbf{Q}^{n-3}(0)+y_{2} \frac{\mathrm{d}}{\mathrm{d} y} \mathbf{Q}^{n-3}(0) \\
& \left.\ldots+y_{n-2} \frac{\mathrm{d}}{\mathrm{d} y} \mathbf{Q}(0)\right\}\left.\right|_{\epsilon=0} \mathbf{u} .
\end{aligned}
$$

The following expansion includes all terms up to fourth order in the small parameter $\epsilon$,

$$
\begin{aligned}
y & =\epsilon \mathbf{u}^{t} \mathbf{u}+\epsilon^{2} \mathbf{u}^{t} \mathbf{Q}_{0} \mathbf{u} \\
& +\epsilon^{3}\left[\mathbf{u}^{t} \mathbf{Q}_{0}^{2} \mathbf{u}+\left(\mathbf{u}^{t} \mathbf{u}\right) \mathbf{u}^{t} \mathbf{Q}_{0}^{\prime} \mathbf{u}\right] \\
& +\epsilon^{4}\left[\mathbf{u}^{t} \mathbf{Q}_{0}^{3} \mathbf{u}+\left(\mathbf{u}^{t} \mathbf{Q}_{0} \mathbf{u}\right) \mathbf{u}^{t} \mathbf{Q}_{0}^{\prime} \mathbf{u}\right. \\
& \left.+2\left(\mathbf{u}^{t} \mathbf{u}\right) \mathbf{u}^{t} \mathbf{Q}_{0} \mathbf{Q}_{0}^{\prime} \mathbf{u}+\frac{1}{2}\left(\mathbf{u}^{t} \mathbf{u}\right)^{2} \mathbf{u}^{t} \mathbf{Q}_{0}^{\prime \prime} \mathbf{u}\right]+\ldots,
\end{aligned}
$$

where

$$
\mathbf{Q}_{0}=\mathbf{Q}(0), \quad \mathbf{Q}_{0}^{\prime}=\mathbf{Q}^{\prime}(0), \quad \mathbf{Q}_{0}^{\prime \prime}=\mathbf{Q}^{\prime \prime}(0) .
$$

The symmetry of $\mathbf{Q}^{t}=\mathbf{Q}$ has been used to simplify terms in the expansion (34).

\section{E. Asymptotic expansion for finite $k b$}

Expanding the function $\mathcal{N}_{p}(\xi b)$ for small $(\xi b-$ $k b)$ yields

$$
\bar{Q}_{0 m n}=D_{m-n}^{(0)}(k b), \quad \bar{Q}_{0 m n}^{\prime}=D_{m-n}^{(1)}(k b),
$$

where

$$
\begin{gathered}
D_{p}^{(0)}(x)=\frac{i \pi}{4}\left[\left(p^{2}-x^{2}\right) J_{p}(x) H_{p}^{(1)}(x)\right. \\
\left.-x^{2} J_{p}^{\prime}(x) H_{p}^{(1)^{\prime}}(x)+2 M_{p}(k b)\right], \\
D_{p}^{(1)}(x)=-\frac{1}{2} D_{p}^{(0)}(x)+\frac{i \pi}{4} M_{p}(k b) \\
+\frac{1}{8}\left[p^{2}-x^{2}-i 2 \pi x^{4} J_{p}(x) H_{p}^{(1)}(x)\right] .
\end{gathered}
$$

Higher derivatives may be found using the identity

$$
\begin{gathered}
(\xi b)^{2} N_{p}^{\prime \prime}+(\xi b) N_{p}^{\prime}+\left((\xi b)^{2}-p^{2}\right) N_{p} \\
=-2(\xi b)^{2} J_{p}(\xi b) H_{p}^{(1)}(k b) .
\end{gathered}
$$

Using the above results, the expansion to $\mathrm{O}\left(\epsilon^{3}\right)$ may be rewritten in terms of the areal density of scatterers, $n_{0}$, as

$$
\xi^{2}=k^{2}+d_{1} n_{0}+d_{2} n_{0}^{2}+d_{3} n_{0}^{3}+\ldots,
$$

where

$$
\begin{aligned}
d_{1} & =-4 i \sum_{n} T_{n}=-4 i f(0) \\
d_{2} & =-\frac{16}{k^{2}} \sum_{m, n} D_{m-n}^{(0)}(k b) T_{m} T_{n}, \\
d_{3} & =\frac{64 i}{k^{4}} \sum_{m, n, p} D_{m-n}^{(0)}(k b) D_{n-p}^{(0)}(k b) T_{m} T_{n} T_{p} \\
& -\frac{16 d_{1}}{k^{4}} \sum_{m, n} D_{m-n}^{(1)}(k b) T_{m} T_{n} .
\end{aligned}
$$

The dependence on $k b$ and the scattering function is contained in the coefficients $d_{1}, d_{2}$, etc.

\section{F. Small $k b$ limit}

In this limit we derive the terms in the series

$$
\xi^{2}=k^{2}+\delta_{1} n_{0}+\delta_{2} n_{0}^{2}+\delta_{3} n_{0}^{3}+\delta_{4} n_{0}^{4}+\ldots
$$

It follows immediately from Eq. (40) that $\delta_{1}=$ $d_{1}$, while $\delta_{2}$ may be found by letting $k b \rightarrow 0$ and using $D_{p}^{(0)}(x)=\frac{|p|}{2}+\mathrm{O}(x)$ and $D_{p}^{(1)}(x)=\frac{p^{2}}{8}-$ $D_{p}^{(0)}(x)+\mathrm{O}(x)$ as $x \rightarrow 0$. It is easier, however, to begin with the small $k b$ expansion of $\overline{\mathbf{Q}}$. This allows us to deduce not only the terms in (39) to $\mathrm{O}\left(n_{0}^{3}\right)$ but the next one. Higher order terms can be found using the procedure described next. As $k b \rightarrow 0$ we have, using (19a),

$$
\mathcal{N}_{p}(\xi b) \simeq \frac{2}{i \pi}\left(\frac{\xi}{k}\right)^{|p|} .
$$

Hence $\overline{\mathbf{Q}}$ becomes independent of $b$,

$$
\bar{Q}_{m n}(\bar{y}) \simeq\left((1+\bar{y})^{\frac{|m-n|}{2}}-1\right) / \bar{y},
$$

from which it follows that

$$
\begin{aligned}
& \bar{Q}_{0 m n}=\frac{1}{2}|m-n|, \\
& \bar{Q}_{0 m n}^{\prime}=\frac{1}{8}|m-n|(|m-n|-2), \\
& \bar{Q}_{0 m n}^{\prime \prime}=\frac{1}{24}|m-n|(|m-n|-2)(|m-n|-4),
\end{aligned}
$$


etc. Now express the asymptotic expansion (34) in terms of the nondimensional parameters $\bar{y}$ and $\bar{\epsilon}$ which do not depend upon $b$,

$$
\begin{aligned}
& \bar{y}=\bar{\epsilon} \operatorname{tr} \mathbf{T}+\bar{\epsilon}^{2} \mathbf{e}^{t} \mathbf{T} \overline{\mathbf{Q}}_{0} \mathbf{T e} \\
& +\bar{\epsilon}^{3}\left[\mathbf{e}^{t} \mathbf{T}\left(\overline{\mathbf{Q}}_{0} \mathbf{T}\right)^{2} \mathbf{e}+(\operatorname{tr} \mathbf{T}) \mathbf{e}^{t} \mathbf{T} \overline{\mathbf{Q}}_{0}^{\prime} \mathbf{T e}\right] \\
& +\bar{\epsilon}^{4}\left[\mathbf{e}^{t} \mathbf{T}\left(\overline{\mathbf{Q}}_{0} \mathbf{T}\right)^{3} \mathbf{e}+\left(\mathbf{e}^{t} \mathbf{T} \overline{\mathbf{Q}}_{0} \mathbf{T e}\right) \mathbf{e}^{t} \mathbf{T} \overline{\mathbf{Q}}_{0}^{\prime} \mathbf{T e}\right. \\
& \left.+2(\operatorname{tr} \mathbf{T}) \mathbf{e}^{t} \mathbf{T} \overline{\mathbf{Q}}_{0} \mathbf{T} \overline{\mathbf{Q}}_{0}^{\prime} \mathbf{T e}+\frac{1}{2}(\operatorname{tr} \mathbf{T})^{2} \mathbf{e}^{t} \mathbf{T} \overline{\mathbf{Q}}_{0}^{\prime \prime} \mathbf{T e}\right] \\
& +\mathrm{O}\left(\bar{\epsilon}^{5}\right)
\end{aligned}
$$

The matrices $\overline{\mathbf{Q}}_{0}, \overline{\mathbf{Q}}_{0}^{\prime}$, etc. are defined in the same way as in (35) for the matrix $\overline{\mathbf{Q}}(\bar{y})$ of (23d). The coefficients in Eq. (41) then follow from eqs. (44) and (45) as

$$
\begin{aligned}
\delta_{1}= & -4 i \sum_{n} T_{n} \\
\delta_{2}= & -\frac{8}{k^{2}} \sum_{m, n}|m-n| T_{m} T_{n} \\
\delta_{3}= & \frac{16 i}{k^{4}} \sum_{m, n, p}|m-n||n-p| T_{m} T_{n} T_{p} \\
& -\frac{2 \delta_{1}}{k^{4}} \sum_{m, n}(m-n)^{2} T_{m} T_{n}-\frac{\delta_{1} \delta_{2}}{2 k^{2}}, \\
\delta_{4}= & \frac{32}{k^{6}} \sum_{m, n, p, q}|m-n||n-p||p-q| T_{m} T_{n} T_{p} T_{q} \\
& +\frac{i 8 \delta_{1}}{k^{6}} \sum_{m, n, p}|m-n|(n-p)^{2} T_{m} T_{n} T_{p} \\
-\sum_{m, n} & {\left[\frac{\delta_{1}^{2}}{3 k^{6}}|m-n|^{3}+\frac{2 \delta_{2}}{k^{4}}(m-n)^{2}\right] T_{m} T_{n} } \\
& -\frac{\delta_{1}^{2} \delta_{2}}{6 k^{4}}-\frac{\delta_{2}^{2}}{2 k^{2}}-\frac{\delta_{1} \delta_{3}}{k^{2}} .
\end{aligned}
$$

\section{G. The Linton-Martin formula generalized}

The coefficients in (46) depend upon the far-field scattering function through its Fourier coefficients. We now show that the coefficients can be expressed in terms of the function $f$ itself rather than its Fourier series. Thus,

$$
\begin{aligned}
& \delta_{1}=-4 i f(0), \\
& \delta_{2}=\frac{8}{\pi k^{2}} \int_{0}^{\pi} \mathrm{d} \theta \cot \left(\frac{\theta}{2}\right) \frac{\mathrm{d}}{\mathrm{d} \theta}[f(\theta) f(-\theta)],
\end{aligned}
$$

$$
\begin{aligned}
& \delta_{3}=\frac{16 i}{\pi^{2} k^{4}} \int_{0}^{\pi} \mathrm{d} \theta \cot \frac{\theta}{2} \int_{0}^{\pi} \mathrm{d} \bar{\theta} \cot \frac{\bar{\theta}}{2} \mathbb{S}(\theta, \bar{\theta}) \\
& +\frac{2 \delta_{1}}{k^{4}} \frac{\mathrm{d}^{2}}{\mathrm{~d} \theta^{2}}[f(\theta) f(-\theta)]_{\theta=0}-\frac{\delta_{1} \delta_{2}}{2 k^{2}}, \\
& \delta_{4}=-\frac{32}{\pi^{3} k^{6}} \int_{0}^{\pi} \mathrm{d} \theta \cot \frac{\theta}{2} \int_{0}^{\pi} \mathrm{d} \bar{\theta} \cot \frac{\bar{\theta}}{2} \int_{0}^{\pi} \mathrm{d} \overline{\bar{\theta}} \cot \frac{\bar{\theta}}{2} \\
& \times \mathbb{T}(\theta, \bar{\theta}, \overline{\bar{\theta}})-\left.\frac{i 8 \delta_{1}}{\pi k^{6}} \int_{0}^{\pi} \mathrm{d} \theta \cot \left(\frac{\theta}{2}\right) \frac{\partial}{\partial \bar{\theta}} \mathbb{S}(\theta, \bar{\theta})\right|_{\bar{\theta}=0} \\
& -\frac{\delta_{1}^{2}}{3 \pi k^{6}} \int_{0}^{\pi} \mathrm{d} \theta \cot \left(\frac{\theta}{2}\right) \frac{\mathrm{d}^{3}}{\mathrm{~d} \theta^{3}}[f(\theta) f(-\theta)] \\
& +\frac{2 \delta_{2}}{k^{4}} \frac{\mathrm{d}^{2}}{\mathrm{~d} \theta^{2}}[f(\theta) f(-\theta)]_{\theta=0}-\frac{\delta_{1}^{2} \delta_{2}}{6 k^{4}}-\frac{\delta_{2}^{2}}{2 k^{2}}-\frac{\delta_{1} \delta_{3}}{k^{2}},
\end{aligned}
$$

where

$$
\begin{aligned}
& \mathbb{S}(\theta, \bar{\theta})=\frac{1}{4} \frac{\partial^{2}}{\partial \theta \partial \bar{\theta}}\{ \\
& f(\theta)[f(-\bar{\theta}) f(\bar{\theta}-\theta)-f(\bar{\theta}) f(-\bar{\theta}-\theta)] \\
& +f(-\theta)[f(\bar{\theta}) f(\theta-\bar{\theta})-f(-\bar{\theta}) f(\theta+\bar{\theta})]\}, \\
& \mathbb{T}(\theta, \bar{\theta}, \overline{\bar{\theta}})=\frac{1}{8} \frac{\partial^{3}}{\partial \theta \partial \bar{\theta} \partial \overline{\bar{\theta}}\{} \\
& \quad f(\theta) f(\bar{\theta}-\theta)[f(-\overline{\bar{\theta}}) f(\overline{\bar{\theta}}-\bar{\theta})-f(\overline{\bar{\theta}}) f(-\overline{\bar{\theta}}-\bar{\theta})] \\
& +f(\theta) f(-\bar{\theta}-\theta)[f(\overline{\bar{\theta}}) f(\bar{\theta}-\overline{\bar{\theta}})-f(-\overline{\bar{\theta}}) f(\bar{\theta}+\overline{\bar{\theta}})] \\
& +f(-\theta) f(\bar{\theta}+\theta)[f(\overline{\bar{\theta}}) f(-\bar{\theta}-\overline{\bar{\theta}})-f(-\overline{\bar{\theta}}) f(\overline{\bar{\theta}}-\bar{\theta})] \\
& +f(-\theta) f(\theta-\bar{\theta})[f(-\overline{\bar{\theta}}) f(\bar{\theta}+\overline{\bar{\theta}})-f(\overline{\bar{\theta}}) f(\bar{\theta}-\overline{\bar{\theta}})]\} .
\end{aligned}
$$

We now justify these expressions.

The first identity for $\delta_{1}$ in (47) is obvious from the definition of Eq. (10). Regarding $\delta_{2}$, we note that the product of $f(\theta)$ and $f(-\theta)$ may be written

$$
f(\theta) f(-\theta)=\sum_{n=-\infty}^{\infty} \sum_{s=-\infty}^{\infty} T_{n} T_{s} e^{i(n-s) \theta} .
$$

Interchanging the indices, implies that the double sum is

$$
f(\theta) f(-\theta)=\sum_{n=-\infty}^{\infty} \sum_{s=-\infty}^{\infty} T_{n} T_{s} \cos (n-s) \theta
$$

This identity is equivalent to Eq. (83) of $\frac{6}{6}$ but without the restriction $T_{n}=T_{-n}$ that was assumed there. The formula for $\delta_{2}$ follows from the relation $\underline{17}$ (Eq. 3.612(7))

$$
\frac{1}{\pi} \int_{0}^{\pi} \mathrm{d} \theta \cot \frac{\theta}{2} \sin m \theta=\operatorname{sgn}(m) .
$$


Turning to the coefficient $\delta_{3}$ in (46c), define

$$
\begin{aligned}
S(\theta, \bar{\theta})= & -\sum_{m} \sum_{n} \sum_{p}(m-n)(n-p) \\
& \times T_{m} T_{n} T_{p} e^{i(m-n) \theta} e^{i(n-p) \bar{\theta}} \\
= & \frac{\partial^{2}}{\partial \theta \partial \bar{\theta}}[f(\theta) f(\bar{\theta}-\theta) f(-\bar{\theta})] .
\end{aligned}
$$

The individual terms in the triple sum are of the form $e^{i \cdots \theta}$, but an identical sum with the preferred dependence $\sin \cdots \theta$ in each term can be obtained by an appropriate permutation of $2^{2}=4$ expressions. The correct combination is

$$
\frac{1}{4}\{S(\theta, \bar{\theta})-S(\theta,-\bar{\theta})-S(-\theta, \bar{\theta})+S(-\theta,-\bar{\theta})\},
$$

which reduces to $\mathbb{S}(\theta, \bar{\theta})$ as given. It may then be deduced, again using the identity (50), that

$$
\begin{aligned}
& \sum_{m, n, p}|m-n||n-p| T_{m} T_{n} T_{p} \\
& \quad=\frac{1}{\pi^{2}} \int_{0}^{\pi} \mathrm{d} \theta \cot \frac{\theta}{2} \int_{0}^{\pi} \mathrm{d} \bar{\theta} \cot \frac{\bar{\theta}}{2} \mathbb{S}(\theta, \bar{\theta}) .
\end{aligned}
$$

The second term in (47c) follows directly from (49). Regarding the coefficient $\delta_{4}$, the third term in the right member of (47d) follows in the same manner as the integral for $\delta_{2}$, based on Eq. (49). The second term in (47d) may be deduced using the relation

$$
\begin{gathered}
\frac{\partial}{\partial \bar{\theta}} \mathbb{S}(\theta, \bar{\theta})=-\sum_{m} \sum_{n} \sum_{p}(m-n)(n-p)^{2} \\
\times T_{m} T_{n} T_{p} \sin (m-n) \theta \cos (n-p) \bar{\theta}
\end{gathered}
$$

evaluated at $\bar{\theta}=0$ in combination with the integral identity (50). Note that

$$
\begin{aligned}
& \left.\frac{\partial}{\partial \theta} \mathbb{S}(\theta, \bar{\theta})\right|_{\bar{\theta}=0}=f^{\prime \prime}(0) \frac{\mathrm{d}}{\mathrm{d} \theta}[f(\theta) f(-\theta)] \\
& -f^{\prime}(0) \frac{\mathrm{d}}{\mathrm{d} \theta}\left[f(\theta) f^{\prime}(-\theta)+f(-\theta) f^{\prime}(\theta)\right] \\
& +f(0) \frac{\mathrm{d}}{\mathrm{d} \theta}\left[f^{\prime}(\theta) f^{\prime}(-\theta)\right]+\frac{f(0)}{2} \frac{\mathrm{d}^{3}}{\mathrm{~d} \theta^{3}}[f(\theta) f(-\theta)] .
\end{aligned}
$$

Finally, the first term in the right member of 477d) may be obtained using the same type of argument used for the identity (52). The function analogous to $S$ now has three arguments,

$$
\begin{gathered}
T(\theta, \bar{\theta}, \overline{\bar{\theta}})=-i \sum_{m} \sum_{n} \sum_{p} \sum_{q}(m-n)(n-p) \\
\times(p-q) T_{m} T_{n} T_{p} T_{q} e^{i(m-n) \theta} e^{i(n-p) \bar{\theta}} e^{i(p-q) \overline{\bar{\theta}}} \\
=\frac{\partial^{3}}{\partial \theta \partial \bar{\theta} \partial \overline{\bar{\theta}}}[f(\theta) f(\bar{\theta}-\theta) f(\overline{\bar{\theta}}-\bar{\theta}) f(-\overline{\bar{\theta}})] .
\end{gathered}
$$

and $2^{3}=8$ permutations are required in order to arrive at the correct quadruple summation, yielding $\mathbb{T}(\theta, \bar{\theta}, \overline{\bar{\theta}})$.

\section{SIMPLIFICATIONS BASED ON THE FINITE RANK OF Q}

The infinite matrix $\mathbf{Q}$ is in practice well approximated by a matrix of finite rank. This follows from the fact that the far-field scattering function $f(\theta)$ is an entire function of the angular argument $\theta$ considered as a complex variable $\underline{18}$. The scattering operator (matrix) $\mathbf{T}$ is therefore compact and has only a finite number of eigenvalues of finite size. At low frequency only the first few elements $T_{m}$ for $m$ near zero are important (monopole, dipole, etc.). Furthermore, as we will see in this section, $\mathbf{Q}$ is of rank 2 in the high frequency limit. Therefore, at any finite frequency the infinite system of equations is really not so in practice, and may be replaced by a finite system. We first develop the solution for finite rank $n$, and then apply it to two important cases; the low frequency Rayleigh limit $(n=3)$ and the high frequency limit $(n=2)$. For the remainder of the paper we take $h$, introduced in Eq. (16), to be zero, so that $M_{p}=0$.

\section{A. $\mathbf{Q}$ is of rank $n$}

As noted above only a finite number of the elements $T_{n}$ are significant at any given frequency. If only $n$ are non-zero, then $\mathbf{Q}$ is of rank $n$. The matrix satisfies an homogeneous equation of degree $n+1$,

$$
\mathbf{Q}^{n+1}+b_{n} \mathbf{Q}^{n}+b_{n-1} \mathbf{Q}^{n-1}+\ldots+b_{1} \mathbf{Q}=0,
$$

with $b_{1}=(-1)^{n}(\operatorname{det} \mathbf{Q}), \cdots, b_{n}=-(\operatorname{tr} \mathbf{Q})$, and therefore,

$$
(\mathbf{I}-\epsilon \mathbf{Q})^{-1}=\mathbf{I}+\beta_{1} \epsilon \mathbf{Q}+\beta_{2} \epsilon^{2} \mathbf{Q}^{2}+\ldots+\beta_{n} \epsilon^{n} \mathbf{Q}^{n},
$$

where

$$
\beta_{j}=\frac{1+\sum_{k=j+1}^{n} b_{k} \epsilon^{n+1-k}}{1+\sum_{m=1}^{n} b_{m} \epsilon^{n+1-m}} .
$$

Thus, using the fundamental result (26) it follows that $y=\left(\xi^{2}-k^{2}\right) b^{2}$ is given by

$$
y=\epsilon \mathbf{u}^{t} \mathbf{u}+\sum_{j=1}^{n} \epsilon^{j+1} \beta_{j} \mathbf{u}^{t} \mathbf{Q}^{j} \mathbf{u} .
$$


Since $\mathbf{Q}=\mathbf{Q}(y)$, (57) is an implicit equation for $y$ which could be solved by iteration, for instance. It may also be written as

$$
\begin{aligned}
\xi^{2}= & k^{2}-4 i n_{0} f(0) \\
& -16 \frac{n_{0}^{2}}{k^{2}} \sum_{j=1}^{n} \bar{\epsilon}^{j-1} \beta_{j} \mathbf{e}^{t}(\mathbf{T} \overline{\mathbf{Q}})^{j} \mathbf{T e} .
\end{aligned}
$$

\section{Rayleigh limit, $\mathbf{Q}$ is of rank 3}

An important case is $n=3$ which is useful at low frequency (Rayleigh limit) when the scattering matrix is well approximated by only three terms: $T_{0}$ and $T_{ \pm 1}$. For $n=3$,

$$
\mathbf{Q}^{4}-\mathrm{I}_{Q} \mathbf{Q}^{3}+\mathbb{I}_{Q} \mathbf{Q}^{2}-\mathbb{I}_{Q} \mathbf{Q}=0,
$$

with $\mathrm{I}_{Q}=\operatorname{tr} \mathbf{Q}, \mathbb{I}_{Q}=\frac{1}{2}\left[(\operatorname{tr} \mathbf{Q})^{2}-\operatorname{tr} \mathbf{Q}^{2}\right], \mathbb{I}_{Q}=$ $\operatorname{det} \mathbf{Q}$. In this case the implicit equation for $y$ becomes

$$
\begin{aligned}
y= & \epsilon \mathbf{u}^{t} \mathbf{u}+\epsilon^{2}\left[\left(1-\epsilon \mathrm{I}_{Q}+\varepsilon^{2} \mathbb{I}_{Q}\right) \mathbf{u}^{t} \mathbf{Q u}\right. \\
& \left.+\left(1-\varepsilon \mathrm{I}_{Q}\right) \epsilon \mathbf{u}^{t} \mathbf{Q}^{2} \mathbf{u}+\epsilon^{2} \mathbf{u}^{t} \mathbf{Q}^{3} \mathbf{u}\right] \\
& \times\left[1-\epsilon \mathrm{I}_{Q}+\varepsilon^{2} \mathbb{I}_{Q}-\epsilon^{3} \mathbb{I}_{Q}\right]^{-1} .
\end{aligned}
$$

More detailed results for the Rayleigh limit are presented in $\$ \mathrm{~V}$ for the particular case of circularly cylindrical scatterers.

\section{B. High frequency limit}

In the high frequency limit $k b, \xi b \gg 1$, we have, from 19a),

$$
\frac{i \pi}{2} N_{p}(\xi b) \underset{(\xi b \rightarrow \infty)}{=} A+(-1)^{p} B,
$$

with

$$
A=\frac{(k+\xi)}{2 \sqrt{k \xi}} e^{i(k-\xi) b}, B=\frac{(k-\xi)}{i 2 \sqrt{k \xi}} e^{i(k+\xi) b} .
$$

The high frequency limit for elastic waves was discussed in ${ }^{8}$, and the same methods developed there could be used for the acoustic problem. It is instructive to note that (61) and (22e), combined with $M_{p}=0$, implies that

$$
\mathbf{Q}=\alpha \mathbf{u} \mathbf{u}^{t}+\beta \mathbf{v} \mathbf{v}^{t},
$$

where

$$
\begin{aligned}
& \alpha=(A-1) / y, \quad \beta=B / y, \\
& \mathbf{v}=\mathbf{T}^{1 / 2}(\ldots,-1,1,-1,, 1, \ldots)^{t},
\end{aligned}
$$

with $v_{0}=T_{0}^{1 / 2}$. The matrix $\mathbf{Q}$ is therefore rank 2 , and the wavenumber $\xi$ can be found using the methods described above.

Equation (60) reduces for rank 2 to

$$
y=\epsilon \mathbf{u}^{t} \mathbf{u}+\epsilon^{2} \frac{(1-\epsilon \operatorname{tr} \mathbf{Q}) \mathbf{u}^{t} \mathbf{Q u}+\epsilon \mathbf{u}^{t} \mathbf{Q}^{2} \mathbf{u}}{1-\epsilon \operatorname{tr} \mathbf{Q}+\varepsilon^{2} \operatorname{det} \mathbf{Q}} .
$$

Noting that

$$
\mathbf{u}^{t} \mathbf{u}=\mathbf{v}^{t} \mathbf{v}=f(0), \quad \mathbf{u}^{t} \mathbf{v}=\mathbf{v}^{t} \mathbf{u}=f(\pi)
$$

and hence

$$
\begin{aligned}
\operatorname{tr} \mathbf{Q} & =(\alpha+\beta) f(0), \\
\operatorname{det} \mathbf{Q} & =\alpha \beta\left(f^{2}(0)-f^{2}(\pi)\right),
\end{aligned}
$$

the equation for $\xi$ becomes

$$
\begin{aligned}
& y=\epsilon f(0)+\epsilon^{2} \times \\
& \left\{\alpha f^{2}(0)+\beta f^{2}(\pi)-\epsilon f(0) \alpha \beta\left(f^{2}(0)-f^{2}(\pi)\right)\right\} \\
& /\left\{1-\epsilon f(0)(\alpha+\beta)+\epsilon^{2} \alpha \beta\left(f^{2}(0)-f^{2}(\pi)\right)\right\} .
\end{aligned}
$$

This simplifies to

$$
\begin{gathered}
\left(\xi^{2}-k^{2}\right)^{2}+4 i n_{0}\left(\xi^{2}-k^{2}\right)(A+B) f(0) \\
-16 n_{0}^{2} A B\left(f^{2}(0)-f^{2}(\pi)\right)=0,
\end{gathered}
$$

or dividing out the factor $\left(\xi^{2}-k^{2}\right)$ (corresponding to the trivial solution $\xi^{2}=k^{2}$ ) implies that $\xi$ at high frequency satisfies

$$
\begin{aligned}
\xi^{2}= & k^{2}-\frac{2 i n_{0}}{\sqrt{\xi k}} f(0)\left[(k+\xi) e^{-i \xi b}+i(\xi-k) e^{i \xi b}\right] e^{i k b} \\
& +\frac{4 i n_{0}^{2}}{\xi k}\left(f^{2}(0)-f^{2}(\pi)\right) e^{i 2 k b} .
\end{aligned}
$$

\section{GENERALIZED SELF CONSISTENT MODEL}

The Generalized Self Consistent Model (GSCM) developed by Yang and $\mathrm{Mal}^{13}$ was derived using a self consistent scheme applied to the Waterman and Truell's formula ${ }^{2}$. Among the objectives of this section is to apply this scheme, which is very broad in scope, to the generalized Linton and Martin formula.

The basic idea follows Christensen and Lo $\stackrel{19}{ }$. Instead of considering cylinders that are directly immersed in fluid, a "three phase cylinder" model is used which assumes that each cylinder is surrounded by a cylindrical ring of fluid, the whole being immersed in a outer region of equivalent fluid 
of unlimited extent. Hence, form functions in this section correspond to three phase cylinders, and, as for cylinders, they may be expressed as a modal sum and calculated numerically 20 .

Let $a$ be the radius of circular cylinders and $c$ $(0 \leq c<1)$ their concentration, the radius $a_{c}$ of cylindrical rings is related to $a$ and $c$ by

$$
c=\frac{n_{0} \pi a^{2}}{n_{0} \pi a_{c}^{2}}=\frac{a^{2}}{a_{c}^{2}} .
$$

Let $\rho_{\text {eff }}$ and $k_{\text {eff }}$ be the effective properties of the equivalent outer fluid. The mass density $\rho_{\text {eff }}$ is defined as the spatial average

$$
\rho_{e f f}=c \rho_{c y l}+(1-c) \rho_{\text {fluid }},
$$

with $\rho_{\text {cyl }}$ and $\rho_{\text {fluid }}$ the cylinder and fluid mass densities. The wave number $k_{e f f}$, which is unknown, is determined with the use of the self consistent scheme.

Without loss of generality, consider the Linton and Martin formula at the second order in concentration. Let $\xi_{L M}$ be the Linton and Martin's effective wave number in the outer equivalent fluid, then we have

$$
\begin{aligned}
\xi_{L M}^{2}= & k_{e f f}^{2}-4 i n_{0} f\left(k_{e f f}, 0\right)+\frac{8 n_{0}^{2}}{\pi k_{e f f}^{2}} \int_{0}^{\pi} \mathrm{d} \theta \\
& \times \cot \left(\frac{\theta}{2}\right) \frac{\mathrm{d}}{\mathrm{d} \theta}\left[f\left(k_{e f f}, \theta\right) f\left(k_{e f f},-\theta\right)\right],
\end{aligned}
$$

with

$$
f\left(k_{e f f}, \theta\right)=\sum_{n} T_{n}\left(k_{e f f}\right) \mathrm{e}^{i n \theta}
$$

and $^{20} n_{0}=c / \pi a^{2}$. The self consistent scheme consists in assuming that $k_{\text {eff }}=\xi_{L M}$. From a physical point of view this means that the outer equivalent fluid is a medium in which the waves propagate in exactly the same manner as the coherent waves. Because $\xi_{L M}=k_{\text {eff }}$ there is no scattering due to the three phase cylinders in the outer equivalent fluid, and the medium can be considered as homogenized. It follows from (69) that $k_{\text {eff }}$ is given by the equation

$$
\begin{aligned}
f\left(k_{e f f}, 0\right)= & \frac{2 n_{0}}{i \pi k_{e f f}^{2}} \int_{0}^{\pi} \mathrm{d} \theta \cot \left(\frac{\theta}{2}\right) \\
& \times \frac{\mathrm{d}}{\mathrm{d} \theta}\left[f\left(k_{e f f}, \theta\right) f\left(k_{e f f},-\theta\right)\right] .
\end{aligned}
$$

It is worth mentioning that at low concentration of cylinders the second term in Eq. (71) can be neglected, so that Eq. (71) reduces to

$$
f\left(k_{e f f}, 0\right)=0,
$$

which corresponds to the equation for the $\mathrm{CPA}^{12}$. In other words, the CPA appears as the approximation of Eq. (71) to first order in concentration.

Another way of presenting the self consistent scheme is to use an iterative procedure applied to Eq. 69). Starting with $k_{0}=k$ we carry out the homogenization by employing Eq. (69) to obtain $k_{1}$, and so on, so that

$$
\begin{aligned}
& k_{n+1}^{2}=k_{n}^{2}-4 i n_{0} f\left(k_{n}, 0\right) \\
& +\frac{8 n_{0}^{2}}{\pi k_{n}^{2}} \int_{0}^{\pi} \mathrm{d} \theta \cot \left(\frac{\theta}{2}\right) \frac{\mathrm{d}}{\mathrm{d} \theta}\left[f\left(k_{n}, \theta\right) f\left(k_{n},-\theta\right)\right] .
\end{aligned}
$$

The iteration is repeated until there is convergence, $k_{n+1} \rightarrow k_{e f f}$, the solution of (71). This procedure is of interest not only for computations but also for its physical interpretation. The effective wave number $k_{1}$ corresponds to a coherent wave that accounts for the double interactions between cylinders according to the basic hypothesis of the Quasi Crystalline Approximation $(\mathrm{QCA})^{2}$. In the same way, $k_{2}$ involves double interactions in the medium characterized by $k_{1}$, the medium in which double interactions are already taken into account. So, relative to the fluid medium characterized by $k_{0}, k_{2}$ must deal with interactions of higher order, even if we do not know how to describe these explicitly. In addition, the number of interactions between cylinders is linked to the order of convergence of the scheme. Accordingly, it can be supposed that the use of the self consistent scheme applied to an explicit effective wavenumber, whatever it is, $k_{I S A}, k_{W T}$ or $k_{L M}$ (whatever the order in concentration), can improve the accuracy of the results while the concentration of cylinders increases. This is what has been observed in 13 when searching for dynamic effective mechanical properties of composites at low frequencies. Furthermore, just as the Linton and Martin approach is more efficient than the one of Waterman and Truell, Eq. (71) should be more efficient than the GSCM introduced by Yang and Mal, and also the CPA. Numerical calculations are beyond the scope of this paper, but the different effective wavenumbers are compared at low frequency in the next section.

\section{THE RAYLEIGH LIMIT}

In the Rayleigh or low-frequency limit, the size of the scatterers is assumed to be small compared to the incident wavelength. In this section scatterers are specifically considered to be circular fluid 
cylinders of radius $a$, characterized by the density $\rho_{2}$ and the sound speed $c_{2}$. They are immersed in a fluid, characterized by the density $\rho_{1}$ and the sound speed $c_{1}$. The Rayleigh limit then corresponds to $k_{1} a \rightarrow 0$ with $k_{1}=\omega / c_{1}$. It is then sufficient to take only the lowest order coefficients in the modal series 21 . More exactly, it can be shown that at low frequencies, only $T_{0}$ and $T_{ \pm 1}$ make a contribution, reducing the infinite matrix $\mathbf{Q}$ to a rank 3 matrix, see \$III.

The goal of this section is to compare the Rayleigh limit for the different effective wave numbers which correspond respectively to ISA, Waterman and Truell, Linton and Martin, CPA, the GSCM developed by Yang and Mal ${ }^{13}$, noted here G-WT, and the GSCM introduced in $\$ \mathrm{IV}$ from the Linton and Martin approach, noted here G-LM.

\section{A. Effective wave numbers}

We first calculate effective wave numbers using the Rayleigh limit of the far-field scattering function associated with cylinders which are directly immersed in fluid:

$$
\begin{aligned}
T_{0} & =\frac{i \pi}{4}\left(\frac{\rho_{1} c_{1}^{2}}{\rho_{2} c_{2}^{2}}-1\right)\left(k_{1} a\right)^{2}, \\
T_{ \pm 1} & =\frac{i \pi}{4}\left(\frac{\rho_{2}-\rho_{1}}{\rho_{2}+\rho_{1}}\right)\left(k_{1} a\right)^{2} .
\end{aligned}
$$

These coefficients can be derived from those corresponding to circular elastic cylinders immersed in fluid ${ }^{21}$, if the shear and longitudinal velocities are equal to 0 and $c_{2}$ respectively (Scattering coefficients are denoted by $R$ in Ref ${ }^{21}$ with $R_{0}=T_{0}$ and $\left.R_{ \pm 1}=-T_{ \pm 1}\right)$.

The use of Eqs.(73) leads to the following results for the ISA, Waterman and Truell (WT) and Linton and Martin (LM). First

$$
\begin{aligned}
\left(\frac{k_{e f f}}{k_{1}}\right)^{2} & =1+\left(\frac{\rho_{1} c_{1}^{2}}{\rho_{2} c_{2}^{2}}+\frac{\rho_{2}-3 \rho_{1}}{\rho_{1}+\rho_{2}}\right) c \\
& \equiv\left(\frac{k_{e f f}}{k_{1}}\right)_{I S A}^{2} \quad \text { (ISA), }
\end{aligned}
$$

in terms of which the other two are

$$
\begin{aligned}
& \left(\frac{k_{e f f}}{k_{1}}\right)^{2}=\left(\frac{k_{e f f}}{k_{1}}\right)_{I S A}^{2}+2 c^{2}\left(\frac{\rho_{2}-\rho_{1}}{\rho_{2}+\rho_{1}}\right) \\
& \times\left\{\begin{array}{l}
\left(\frac{\rho_{1} c_{1}^{2}}{\rho_{2} c_{2}^{2}}-1\right) \\
\left(\frac{\rho_{1} c_{1}^{2}}{\rho_{2} c_{2}^{2}}-\frac{2 \rho_{1}}{\rho_{2}+\rho_{1}}\right)
\end{array}\right.
\end{aligned}
$$

Note that the latter two effective wavenumbers are almost the same if the densities $\rho_{1}$ and $\rho_{2}$ are close to one another in value. However, even at low frequency where cylinders look like "point scatterers", the Waterman and Truell and the Linton and Martin approaches give different results as soon as $\rho_{2}$ is not close to $\rho_{1}$.

\section{B. Wave numbers from the self consistent scheme}

We now consider effective wave numbers obtained by the self consistent scheme, which uses scattering coefficients for the "three phase cylinder" as described in \$IV] The coefficients are calculated as outlined in ${ }^{22}$, with the results

$$
\begin{aligned}
& T_{0}=\frac{i \pi}{4}\left((1-c) \frac{\rho_{\text {eff }}}{\rho_{1}}+c \frac{\rho_{\text {eff }} c_{1}^{2}}{\rho_{2} c_{2}^{2}}-\frac{k_{\text {eff }}^{2}}{k_{1}^{2}}\right)\left(k_{1} a_{c}\right)^{2}, \\
& T_{ \pm 1}=\frac{i \pi}{4} F\left(\rho_{\text {eff }}\right)\left(k_{\text {eff }} a_{c}\right)^{2},
\end{aligned}
$$

where $\rho_{\text {eff }}=(1-c) \rho_{1}+c \rho_{2}$ from Eq. (68), and

$$
\begin{aligned}
& \quad F\left(\rho_{e f f}\right)= \\
& \frac{(1-c)\left(\rho_{1}^{2}-\rho_{2} \rho_{e f f}\right)+(1+c) \rho_{1}\left(\rho_{2}-\rho_{e f f}\right)}{(1-c)\left(\rho_{1}^{2}+\rho_{2} \rho_{e f f}\right)+(1+c) \rho_{1}\left(\rho_{2}+\rho_{e f f}\right)} .
\end{aligned}
$$

Note that Eqs. (73) follow from Eqs. (76) formally if we first put $k_{\text {eff }}=k_{1}$ and $\rho_{\text {eff }}=\rho_{1}$, in order to identify the outer equivalent fluid with the fluid itself, and then cancel the ring of fluid by setting $a_{c}=a$, which implies $c=1$.

We now consider three approaches based on self consistent schemes: the CPA, the generalized self consistent method based on Waterman and Truell (G-WT), and the same for Linton and Martin's approach (G-LM). We find

$$
\begin{aligned}
& \left(\frac{k_{e f f}}{k_{1}}\right)^{2}=\frac{\rho_{e f f}}{\rho_{1}}\left[1+\left(\frac{\rho_{1} c_{1}^{2}}{\rho_{2} c_{2}^{2}}-1\right) c\right] \\
& \quad \times \begin{cases}{\left[1-2 F\left(\rho_{e f f}\right)\right]^{-1}} & (\mathrm{CPA}), \\
{\left[1+2 F\left(\rho_{e f f}\right)\right]} & (\mathrm{G}-\mathrm{WT}), \\
{\left[\frac{1+2 F\left(\rho_{e f f}\right)}{1-2 F^{2}\left(\rho_{e f f}\right)}\right]} & (\mathrm{G}-\mathrm{LM}) .\end{cases}
\end{aligned}
$$

In order to compare these effective wave numbers with those of eqs. (74) and (75) in the previous subsection, we have to perform an asymptotic expansion with regard to the concentration of scatterers. It follows that

$$
F\left(\rho_{e f f}\right)=\frac{\left(\rho_{1}-\rho_{2}\right)^{2}}{2 \rho_{1}}\left\{\frac{-c}{\rho_{1}+\rho_{2}}+\frac{c^{2}}{2 \rho_{1}}+\ldots\right\},
$$

Multiple scattering by cylinders 10 
and we find at the second order in concentration

$$
\begin{aligned}
& \left(\frac{k_{e f f}}{k_{1}}\right)^{2}=\left(\frac{k_{e f f}}{k_{1}}\right)_{I S A}^{2}+2 c^{2}\left(\frac{\rho_{2}-\rho_{1}}{\rho_{2}+\rho_{1}}\right) \\
& \times \begin{cases}{\left[\frac{\rho_{1} c_{1}^{2}}{\rho_{2} c_{2}^{2}}-\frac{2 \rho_{1}}{\rho_{2}+\rho_{1}}-\frac{\left(\rho_{1}-\rho_{2}\right)^{2}}{4 \rho_{1}\left(\rho_{1}+\rho_{2}\right)}\right]} & (\mathrm{CPA}), \\
{\left[\frac{\rho_{1} c_{1}^{2}}{\rho_{2} c_{2}^{2}}-\frac{2 \rho_{1}}{\rho_{2}+\rho_{1}}+\frac{\left(\rho_{1}-\rho_{2}\right)^{2}}{4 \rho_{1}\left(\rho_{1}+\rho_{2}\right)}\right]} & (\mathrm{G}-\mathrm{WT}), \\
{\left[\frac{\rho_{1} c_{1}^{2}}{\rho_{2} c_{2}^{2}}-\frac{2 \rho_{1}}{\rho_{2}+\rho_{1}}\right]} & (\mathrm{G}-\mathrm{LM})\end{cases}
\end{aligned}
$$

As expected, all three methods give the same result at the first order in concentration. At the second order, the CPA and G-WT introduce the same additional term as compared to Linton and Martin's approach, but with the opposite sign in each. It is significant that the self consistent scheme applied to Linton and Martin's formula does not modify the result, at least at this order. The same cannot be said of the CPA and the Waterman and Truell methods. Thus, we may conclude that Linton and Martin's approach and the G-LM can be considered "self consistent" methods. Finally, we note that the additional terms in the CPA and in the G-WT in Eq. (78) are very small if the densities $\rho_{1}$ and $\rho_{2}$ are close in value. Hence, all the methods are equivalent at low frequency when the densities $\rho_{1}$ and $\rho_{2}$ are equal. Of course, these results say nothing about what happens at higher frequency and at higher concentration.

\section{CONCLUSIONS}

Implications of the quasi-crystalline approximation (QCA) on the effective wave number beyond the dilute concentration limit have been described. Equation (28) is the starting point for all further developments, and as such represents the fundamental result of the paper. It splits the implicit form of the effective wavenumber into two distinct parts, one defined by the single scatter T-matrix, $\mathbf{T}$, and the other by the spatial arrangement of the scatterers, $\overline{\mathbf{Q}}$. In this paper we have used to the hole correction, for which $\overline{\mathbf{Q}}$ is given by (23d). More generally, this matrix has elements

$$
\bar{Q}_{m n}=\frac{L_{m-n}}{-i 4 n_{0}} e^{-i \xi x_{1}}+\frac{e^{i(k-\xi) x_{1}}}{2 k(\xi-k)}-\frac{1}{\xi^{2}-k^{2}},
$$

where $L_{m-n}$ is defined by Eq. (15) for arbitrary pair correlation function. The QCA is exact for a regular array of scatterers, in which case $\bar{Q}_{m n}$ can be reduced to a known lattice sum. Equation (28) therefore provides a formula for determining the dispersion curves of a regular array. This and other implications will be examined elsewhere.
${ }^{1}$ L. L. Foldy. The multiple scattering of waves. I. General theory of isotropic scattering by randomly distributed scatterers. Phys. Rev., 67:107119, 1945.

2 P. C. Waterman and R. Truell. Multiple scattering of waves. J. Math. Phys., 2(4):512-537, 1961.

${ }^{3}$ Y. C. Angel and C. Aristégui. Analysis of sound propagation in a fluid through a screen of scatterers. J. Acoust. Soc. Am., 118(1):72-82, 2005.

${ }^{4}$ P. Lloyd and M.V. Berry. Wave propagation through an assembly of spheres. IV. Relation between different scattering theories. Proc. Phys. Soc. London, 91:678-688, 1967.

${ }^{5}$ C. M. Linton and P. A. Martin. Multiple scattering by multiple spheres: a new proof of the Lloydberry formula for the effective wavenumber. SIAM J. Appl. Math., 66(5):1649-1668, 2006.

${ }^{6}$ C. M. Linton and P. A. Martin. Multiple scattering by random configurations of circular cylinders: Second-order corrections for the effective wavenumber. J. Acoust. Soc. Am., 117(6):3413-3423, 2005. doi: 10.1121/1.1904270.

7 J. G. Fikioris and P. C. Waterman. Multiple scattering of waves. II. "Hole corrections" in the scalar case. J. Math. Phys., 5(10):1413-1420, 1964.

8 J. M. Conoir and A. N. Norris. Effective wave numbers and reflection coefficients for an elastic medium containing random configurations of cylindrical scatterer. Wave Motion, 47:183-197, 2010. doi: 10.1016/j.wavemoti.2009.09.004.

${ }^{9}$ P. A. Martin and A. Maurel. Multiple scattering by random configurations of circular cylinders: Weak scattering without closure assumptions. Wave Motion, 45:865-880, 2008. doi: 10.1016/j.wavemoti. 2008.03.004.

10 A. Lagendijk and B. A. Van Tiggelen. Resonant multiple scattering of light. Phys. Rep., 270:143215, 1996.

11 A. Derode, V. Mamou, and A. Tourin. Influence of correlations between scatterers on the attenuation of the coherent wave in a random medium. Phys. Rev. E, 74:036606, 2006.

12 P. Sheng. Introduction to Wave Scattering, Localization, and Mesoscopic Phenomena. Academic Press, San Diego, 1995.

13 R. Yang and A. K. Mal. Multiple scattering of elastic waves in a fiber-reinforced composite. $J$. Mech. Phys. Solids, 42:1945-1968, 1994. doi: http: //dx.doi.org/10.1016/0022-5096(94)90020-5.

14 P. Y. Le Bas, F. Luppé, and J. M. Conoir. Reflection and transmission by randomly spaced elastic cylinders in a fluid slab-like region. J. Acoust. Soc. Am., 117(3):1088-1097, 2005. doi: 10.1121/1.1848174.

15 M. L. Cowan, K. Beaty, J. H.Page, Z. Liu, and P. Sheng. Group velocity of acoustic waves in strongly scattering media: dependence on the volume fraction of scatterers. Phys. Rev. E, 58(5): 6626-6636, 1998.

16 J. Y. Kim. Models for wave propagation in two- 
dimensional random composites: A comparative study. J. Acoust. Soc. Am., 127(4):2201-2209, 2010.

17 I. S. Gradshteyn and I. M. Ryzhik. Table of Integrals, Series, and Products. Page 366. Academic Press: New York, 1980.

18 C. Muller. Radiation patterns and radiation fields. J. Rat. Mech. Anal, 4:235-246, 1955.

19 R.M. Christensen and K.H. Lo. Solutions for effective shear properties in three phase sphere and cylinder models. J. Mech. Phys. Solids, 27:315-330, 1979.

${ }^{20}$ V.K. Varadan and V.V. Varadan. Acoustic, Elec- tromagnetic, and Elastic Waves Scattering-Focused on the T-Matrix Approach. Pergamon Press, NewYork, 693 pages, 1980.

21 J. G. Minonzio, C. Prad, D. Chambers, D. Clorennec, and M. Fink. Characterization of subwavelength elastic cylinders with the decomposition of the time-reversal operator: Theory and experiment. J. Acoust. Soc. Am., 117(2):789-798, 2005.

22 N.D. Veksler. Resonance Acoustic Spectroscopy. Springer-Verlag, Berlin Heidelberg, 282 pages, 1993. 